\title{
Renoprotective effects of emodin against diabetic nephropathy in rat models are mediated via PI3K/Akt/GSK-3 $\beta$ and Bax/caspase-3 signaling pathways
}

\author{
DANQING JING, HUA BAI and SHINAN YIN \\ Department of Endocrinology, The First Affiliated Hospital of PLA General Hospital, Beijing 100048, P.R. China
}

Received April 2, 2016; Accepted March 10, 2017

DOI: 10.3892/etm.2017.5131

\begin{abstract}
Emodin is the main active component of the Chinese medicine rhubarb, which has a variety of pharmacological effects and a high clinical value. Its anti-inflammatory and antitumor effects have been widely studied. The aim of the present study was to determine whether emodin has renoprotective effects, and to identify the potential underlying mechanisms in a rat model of diabetic nephropathy (DN). The changes in mean blood glucose levels, normalized kidney weight, urinary albumin excretion, serum creatinine levels and tubulointerstitial injury index (TII) scores of the rats with DN were significantly attenuated by emodin. Furthermore, treatment with emodin significantly inhibited inflammation-related factors and oxidative stress, suppressed the expression of intercellular adhesion molecule 1 (ICAM-1) and B-cell lymphoma 2-associated X protein (Bax), increased phosphorylated Akt and phosphorylated-glycogen synthase kinase 3 (p-GSK-3 $\beta$ ) expression and inhibited caspase- 3 activity in diabetic rats. These data suggest that emodin protects against DN and that the underlying mechanism may involve the suppression of inflammation, ICAM-1 and Bax, and activation of the PI3K/Akt/GSK-3 $\beta$ pathway.
\end{abstract}

\section{Introduction}

With improvements in the availability of food and changes in lifestyle, the incidence of diabetes has increased significantly. According to an estimate in 2007 by the International Diabetes Federation, the number of people with diabetes in China was 39.8 million, ranking second in the world after India. Furthermore, there was an annual increase of 1.01 million diabetics, among which $95 \%$ had type 2 diabetes (1). Great progress has been made in the diagnosis and prevention of diabetic

Correspondence to: Dr Shinan Yin, Department of Endocrinology, The First Affiliated Hospital of PLA General Hospital, 51 Fucheng Road, Beijing 100048, P.R. China

E-mail: sfseq31@163.com

Key words: emodin, diabetic nephropathy, intercellular adhesion molecule 1, phosphoinositide 3 kinase, Akt, glycogen synthase kinase 3 nephropathy (DN), a common complication of diabetes (2). However, kidney damage in patients with diabetes is not necessarily due to DN (2).

DN pathogenesis is very complex, and involves lipid disorders, hemodynamic abnormalities, the release of inflammatory mediators, cytokines, oxidative stress and apoptosis (2). Recently with development of biotechnological techniques, the research into and awareness of podocytes have gradually increased (3). Furthermore, podocyte injury in the pathogenesis of DN has been recognized as the key factor causing DN proteinuria and glomerular sclerosis, which may prompt the development of new strategies for the prevention and treatment of DN (4).

The phosphoinositide 3 kinase (PI3K)/protein kinase B (also known as Akt) signaling pathway participates in cell differentiation, proliferation, apoptosis and migration, and excessive activation of this pathway leads to cell dysfunction (5). As mentioned above, podocyte injury has been recognized to play a role in DN (6); changes of marker protein expression are the basis of changes in podocyte function, and the PI3K/Akt signaling pathway may change the podocyte phenotype and induce the podocyte injury that is involved in the progression of DN (7).

Glycogen synthase kinase 3 (GSK-3) is a rate-limiting enzyme for inhibiting glycogen synthesis, and is involved in the regulation of various signals, including insulin cell signaling (8). The GSK-3 $\beta$ subtype is primarily involved in this; therefore, research into the correlation between GSK-3 $\beta$ and type 2 diabetes has been conducted (9). Previous studies have shown a correlation between high expression levels of GSK-3 $\beta$ and decreased insulin sensitivity $(8,10)$.

Emodin is an anthraquinone derivative, which may be obtained from the root of Rheum palmatum L. (rhubarb). The anti-inflammatory effect of emodin has been demonstrated in many experiments using animal models (11). Additionally, its antibacterial effects on Pseudomonas aeruginosa and Staphylococcus aureus, methicillin-resistant Staphylococcus aureus have also been reported (12). Emodin has also been shown to inhibit the growth of Helicobacter pylori in vitro in a dose-dependent manner (13). Further in vitro experiments have demonstrated that emodin significantly inhibits the release of cytokines by mononuclear cells under lipopolysaccharide (LPS) stimulation, and also inhibits the endotoxin-induced secretion of tumor necrosis factor (TNF)- $\alpha$, interleukin (IL)-1, 
IL-6, IL-8 and other inflammatory cytokines, thus affecting the immune activation associated with them $(14,15)$.

\section{Materials and methods}

Experimental animals. All experimental procedures were pre-approved by the First Affiliated Hospital of PLA General Hospital (Beijing, China). Female adult Wistar rats (8 weeks old, 200-230 g) were obtained from Beijing Vital River Laboratory Animal Technology Co., Ltd. (Beijing, China) and housed at $21-23^{\circ} \mathrm{C}$ with a $12: 12 \mathrm{~h}$ light-dark cycle and $55-60 \%$ relative humidity. Furthermore, standard pelletized food and water were provided ad libitum. Rats were randomly divided into three groups: Control $(n=6)$; DN model $(n=8)$ and emodin $(n=8)$ groups. The DN model and emodin group rats were administered a $60 \mathrm{mg} / \mathrm{kg}$ intraperitoneal injection of streptozotocin (Sigma-Aldrich, Inc.; Merck KGaA, Darmstadt, Germany). The emodin group rats were administered emodin $100 \mathrm{mg} / \mathrm{kg}$ dose once every 3 days (Sigma-Aldrich, Inc.; Merck KGaA) for 3 weeks.

Normalized kidney weight, glucose urine albumin and creatinine measurements. Following the 3 weeks of treatment with emodin, kidneys were acquired and washed with phosphate-buffered saline (PBS). Next, each kidney was weighed and the normalized kidney weight was determined. Glucose (F006), Urine albumin (A028-1) and creatinine (C011-2) were quantified with commercial kits (Nanjing Jiancheng Biology Engineering Institute, Nanjing, China) and the Cayman creatinine assay kit (Ann Arbor, MI, USA) was also used to determine creatinine levels.

Histological analysis and determination of tubulointerstitial injury index (TII) score. After the 3 weeks of treatment with emodin, kidneys were acquired and washed with PBS. The tissue was then fixed in $4 \%$ formaldehyde for $24 \mathrm{~h}$ and embedded in paraffin. The tissue was cut into $8-\mu \mathrm{m}$ sections and stained using hematoxylin and eosin (Fuzhou Maixin Biotech. Co., Ltd., Fuzhou, China). The stained kidney tissue was then observed using light microscopy (Nikon 80i; Nikon, Tokyo, Japan). Sections were stained with Periodic acid-Schiff assay for $1 \mathrm{~h}$. TII scores was scored as the percentage of tubulointerstitial injury area within the total area: 0 , normal; $1,<25 \% ; 2,25-50 \%$; $3,>50 \%$.

ELISA analysis of inflammatory factors, oxidative stress and caspase-3. Blood was obtained through the inferior vena cava of every rat and centrifuged at $12,000 \mathrm{x}$ g for $10 \mathrm{~min}$ at $4^{\circ} \mathrm{C}$. IL-6, TNF- $\alpha$, superoxide dismutase (SOD), malondialdehyde (MDA) and caspase-3 levels were measured using ELISA kits (Nanjing Keygen Biotech, Co., Ltd., Jiangsu, China).

Western blot analysis. After the 3 weeks of treatment with emodin, kidney tissues were lysed with ice-cold radioimmunoprecipitation assay buffer (Bio-Rad Laboratories, Inc.) A bicinchoninic acid protein assay kit (Beyotime Institute of Biotechnology, Haimen, China) was used to quantify the protein contents. Protein $(30 \mu \mathrm{g})$ was loaded onto a gel for $10 \%$ Tris-glycine-SDS-PAGE and electroblotted onto polyvinylidene difluoride membranes (Bio-Rad Laboratories, Inc.). The

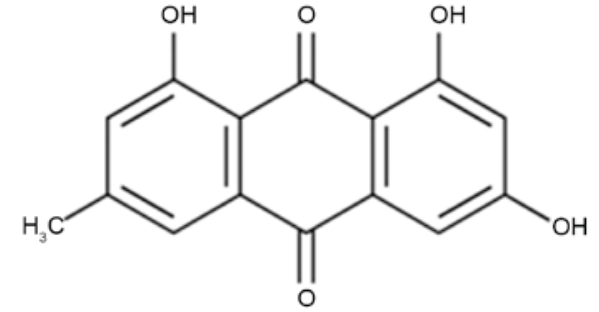

Figure 1. Chemical structure of emodin.

membranes were then blocked with $5 \%$ non-fat milk in PBS $0.05 \%$ Tween 20 solution. Sections were then incubated with specific primary antibodies against intercellular adhesion molecule 1 (ICAM-; sc-7891; 1:500), Bax (sc-6236; 1:500), p-Akt (sc-7985-R; 1:200), Akt (sc-8312; 1:500), p-GSK-3 $\beta$ (sc-135653; 1:200), GSK-3ß (sc-7879; 1:500), caspase-3 (sc-98785; 1:500, Santa Cruz Biotechnology, Inc., Dallas, TX, USA) and GAPDH (sc-25778; 1:2,000; Santa Cruz Biotechnology, Inc.) at $4^{\circ} \mathrm{C}$ overnight. Membranes were then washed with a PBS $0.05 \%$ Tween 20 solution prior to incubation with polyclonal goat anti-mouse horseradish peroxidase-conjugated secondary antibody (sc-2031; 1:5,000; Santa Cruz Biotechnology, Inc.) for $1 \mathrm{~h}$ at $37^{\circ} \mathrm{C}$. The membranes were then detected using a chemiluminescence detection kit (Amersham Pharmacia Biotech; GE Healthcare Life Sciences, Chalfont, UK). Protein expression was quantified using Image J 1.32 software (National Institutes of Health, Bethesda, MD, USA).

Statistical analysis. Values are presented as the mean \pm standard deviation. One-way analysis of variance was used to identify differences among groups using Tukey's tests. $\mathrm{P}<0.05$ was used to indicate a statistically significant difference.

\section{Results}

Blood glucose levels, normalized kidney weight, urinary albumin excretion and serum creatinine levels of diabetic rats. The chemical structure of emodin is shown in Fig. 1. As shown in Fig. 2, there was a significant increase in the blood glucose level, normalized kidney weight, urinary albumin excretion and serum creatinine level in the DN model group compared with the control group. Following treatment with emodin, the increases in blood glucose level, normalized kidney weight, urinary albumin excretion and serum creatinine level were significantly attenuated, compared with those in the DN model group (Fig. 2).

TII scores of diabetic rats. The TII scores of the DN model group were observed to be significantly increased compared with those in the control group (Fig. 3). Notably, treatment with emodin significantly reduced the elevation of the TII scores in the DN model rats (Fig. 3).

Inflammation factors of diabetic rats. The potential anti-inflammatory effects of emodin in rats with DN were investigated via the analysis of IL- 6 and TNF- $\alpha$ levels. The ELISA results presented in Fig. 4 revealed that the IL- 6 and TNF- $\alpha$ levels in the group were significantly increased compared with 

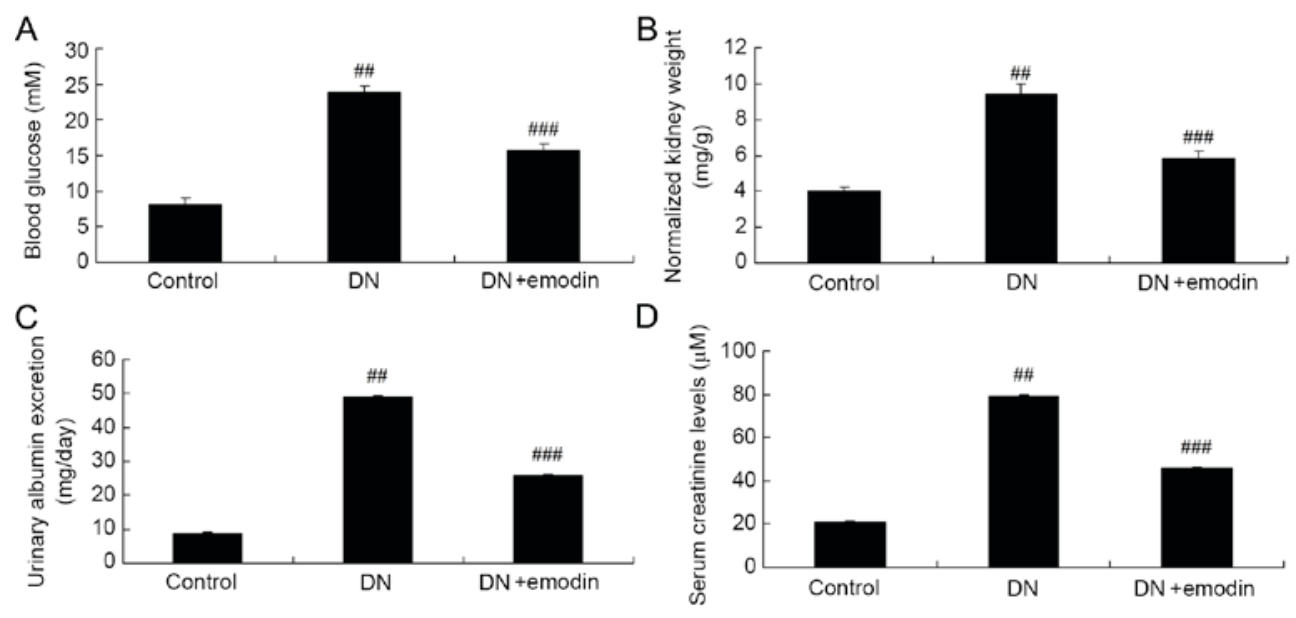

Figure 2. Average blood glucose levels, normalized kidney weight, urinary albumin excretion and serum creatinine levels of diabetic rats. (A) Average blood glucose levels, (B) normalized kidney weight, (C) urinary albumin excretion and (D) serum creatinine levels of diabetic rats. ${ }^{\# \#} \mathrm{P}<0.01 \mathrm{vs.}$. the control group;

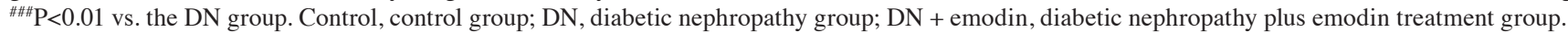

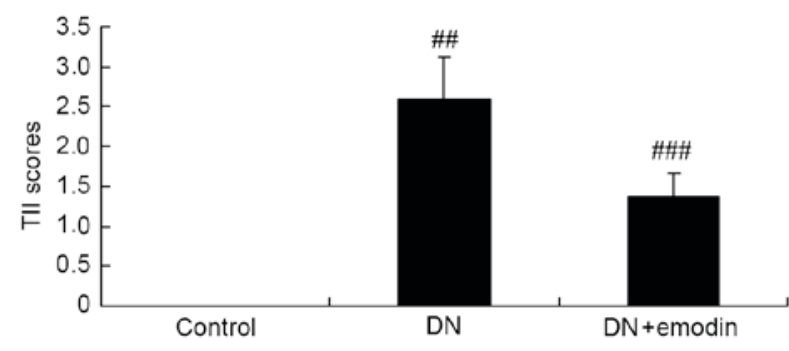

Figure 3. TII scores of diabetic rats. ${ }^{\# \#} \mathrm{P}<0.01$ vs. the control group; ${ }^{\# \#} \mathrm{P}<0.01$ vs. the DM group. Control, control group; DN, diabetic nephropathy group; $\mathrm{DN}+$ emodin, diabetic nephropathy plus emodin treatment group; TII, tubulointerstitial injury index.

those in the control group. Furthermore, comparison of the DN and emodin groups indicated that emodin significantly decreased the activation of IL- 6 and TNF- $\alpha$ levels in rats with DN (Fig. 4).

Oxidative stress of diabetic rats. SOD and MDA levels were analyzed in order to determine whether emodin exhibited an anti-oxidative effect in rats with DN. The results presented in Fig. 5 show that DN significantly reduced the SOD level and increased the MDA level in diabetic rats, compared with the control group. Furthermore, treatment with emodin significantly attenuated the changes in SOD and MDA levels compared with those in the DN model group (Fig. 5.

ICAM-1 protein expression of diabetic rats. ICAM-1 expression was evaluated using western blot analysis in order to investigate whether ICAM-1 pathways are involved in the protective effect of emodin against DN. As shown in Fig. 6, activation of ICAM-1 expression was observed in the DN model group, compared with the control group. The increased ICAM-1 expression in the DN model was significantly suppressed by treatment with emodin (Fig. 6).

Bax protein expression of diabetic rats. Bax expression in the kidneys of diabetic rats was detected by western blot analysis (Fig. 7). DN significantly increased the expression of Bax in the DN model group compared with the control group (Fig. 7). Treatment with emodin significantly reduced the Bax protein expression compared with that in the DN model group (Fig. 7).

p-Akt protein expression of diabetic rats. The effect of emodin on Akt pathways in the kidneys of diabetic rats was also investigated. As shown in Fig. 8, there was a significant inhibition of Akt pathways in the DN model group compared with the control group, as demonstrated by a reduction in the phosphorylation level of Akt. However, emodin significantly promoted the p-Akt protein expression of rats with $\mathrm{DN}$, when compared with the DN model group (Fig. 8).

$p$-GSK-3 $\beta$ protein expression of diabetic rats. p-GSK-3 $\beta$ protein expression was evaluated using western blotting in order to examine whether there is an association between GSK-3 $\beta$ pathways and the protective effect of emodin on DN. The results shown in Fig. 9 indicate that DN significantly inhibited GSK-3 $\beta$ pathways and p-GSK-3 $\beta$ protein expression in diabetic rats compared with the control group, and treatment with emodin significantly activated the protein expression of p-GSK-3 $\beta$ compared with that in the DN model group (Fig. 9).

Caspase- 3 expression of diabetic rats. Caspase- 3 expression in diabetic rats was measured using ELISA and western blotting in order to elucidate the protective effect of emodin on apoptosis in DN rats. Caspase-3 activity and protein expression in the DN model group was significantly increased compared with that in the control group (Fig. 10). However, these changes to caspase-3 in the rats with DN were significantly inhibited by treatment with emodin (Fig. 10).

\section{Discussion}

DN is a complication of diabetes, and the incidence of nephropathy among diabetic patients is much higher than that in non-diabetic patients (16). It is classified as a serious microvascular disease, which can cause kidney damage, and eventually lead to end-stage renal disease. The basic pathological change in DN comprises glomerulosclerosis caused 

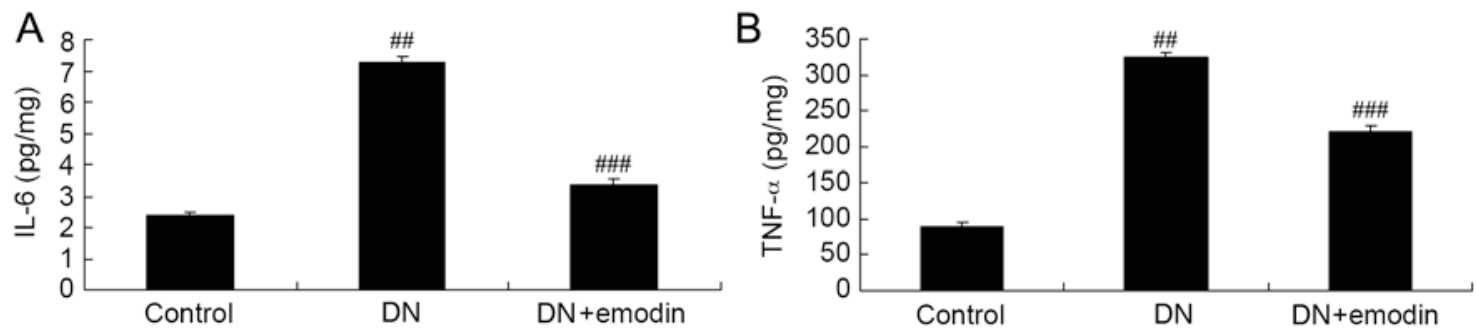

Figure 4. Inflammatory factors of diabetic rats. (A) IL-6 and (B) TNF- $\alpha$ expression in the kidneys of diabetic rats. ${ }^{\# \#} \mathrm{P}<0.01$ vs. the control group; ${ }^{\# \# \#} \mathrm{P}<0.01$ vs. the DM group. Control, control group; DN, diabetic nephropathy group; DN + emodin, diabetic nephropathy plus emodin treatment group; IL, interleukin; TNF- $\alpha$, tumor necrosis factor- $\alpha$.
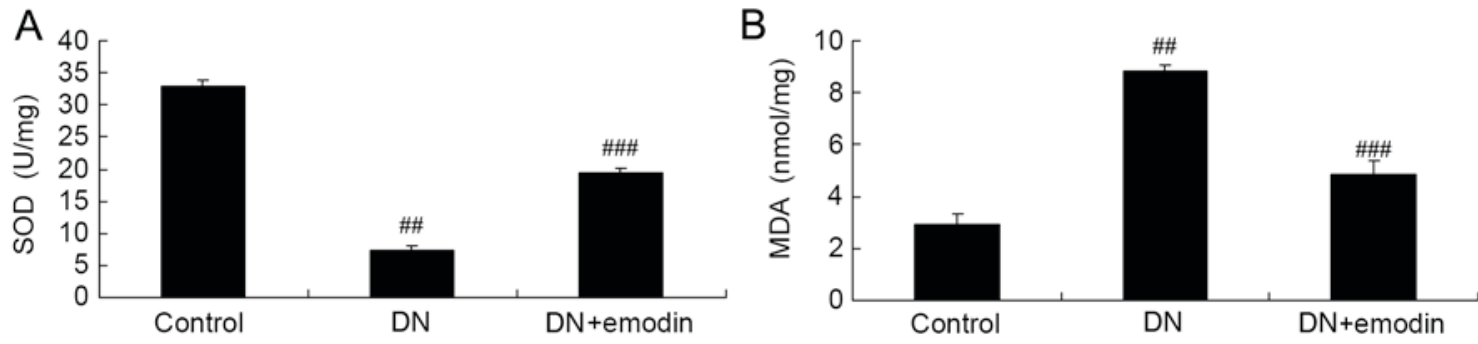

Figure 5. Oxidative stress of diabetic rats. (A) SOD and (B) MDA in the kidneys of diabetic rats. ${ }^{\# \#} \mathrm{P}<0.01$ vs. the control group; ${ }^{\# \# \#} \mathrm{P}<0.01$ vs. the DM group. Control, control group; DN, diabetic nephropathy group; DN + emodin, diabetic nephropathy plus emodin treatment group; SOD, superoxide dismutase; MDA, malondialdehyde.
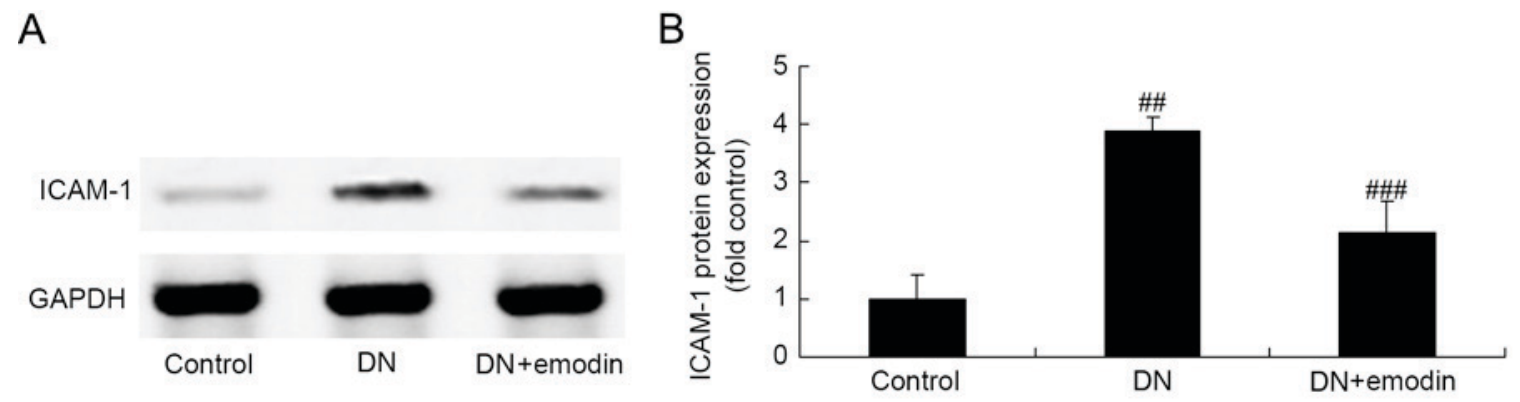

Figure 6. ICAM-1 expression of diabetic rats. (A) Western blot analysis and (B) quantification of ICAM-1 protein expression in the kidneys of diabetic rats. ${ }^{\#} \mathrm{P}<0.01$ vs. the control group; ${ }^{\# \#} \mathrm{P}<0.01$ vs. the DM group. Control, control group; DN, diabetic nephropathy group; DN + emodin, diabetic nephropathy plus emodin treatment group; ICAM-1, intercellular adhesion molecule 1.

A

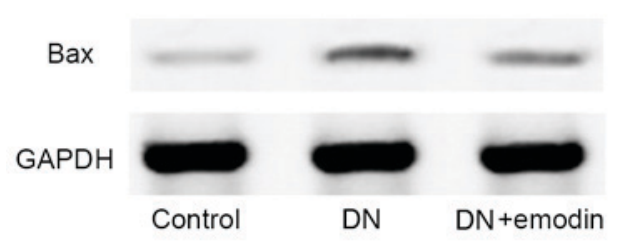

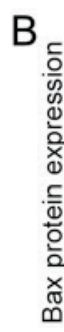

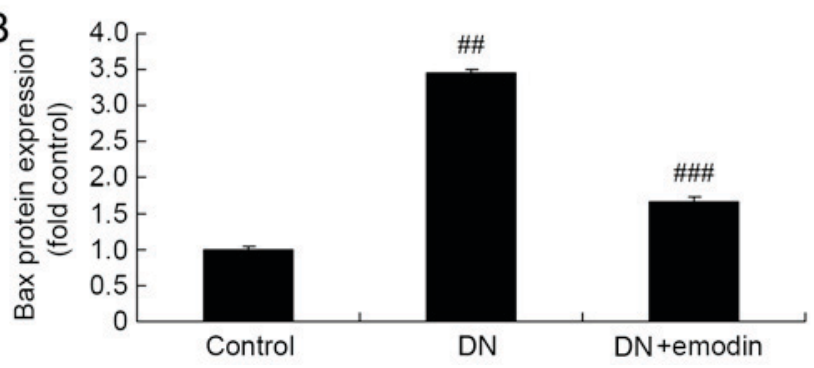

Figure 7. Bax protein expression of diabetic rats. (A) Western blot analysis and (B) quantification of Bax protein expression in the kidneys of diabetic rats. ${ }^{\# \#} \mathrm{P}<0.01$ vs. the control group; ${ }^{\# \# \#} \mathrm{P}<0.01$ vs. the DM group. Control, control group; DN, diabetic nephropathy group; DN + emodin, diabetic nephropathy plus emodin treatment group; Bax, B-cell lymphoma 2 associated $\mathrm{X}$ protein.

by glomerular cell proliferation, the release of inflammatory mediators and accumulation of extracellular matrix and other factors (17). Proteinuria is the clinical manifestation in the earliest stage. DN is not traditionally considered a disease that is associated with the immune system; however, a study has shown that activation of the innate immune system and inflammatory mechanisms are important in its pathogenesis (18). In patients with DN-associated kidney inflammation, 
A

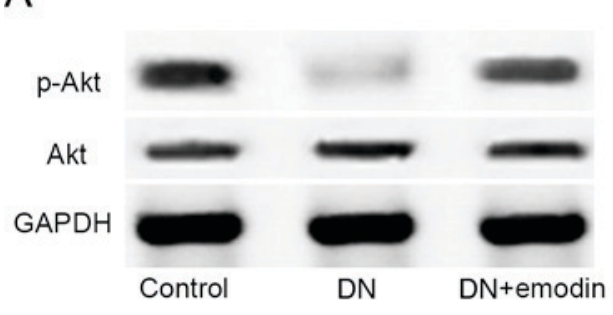

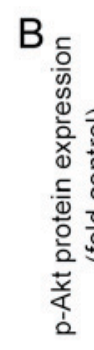

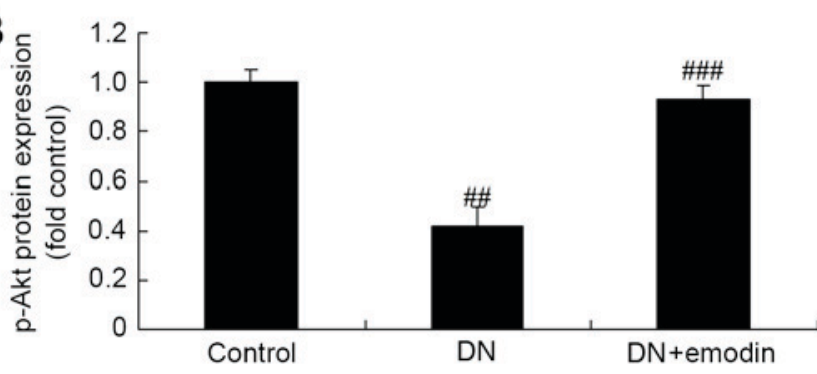

Figure 8. p-Akt protein expression of diabetic rats. (A) Western blot analysis and (B) quantification of p-Akt protein expression in the kidneys of diabetic rats. ${ }^{\# \#} \mathrm{P}<0.01$ vs. the control group; ${ }^{\# \#} \mathrm{P}<0.01$ vs. the $\mathrm{DM}$ group. Control, control group; DN, diabetic nephropathy group; DN + emodin, diabetic nephropathy plus emodin treatment group; p-Akt, phosphorylated Akt.

A

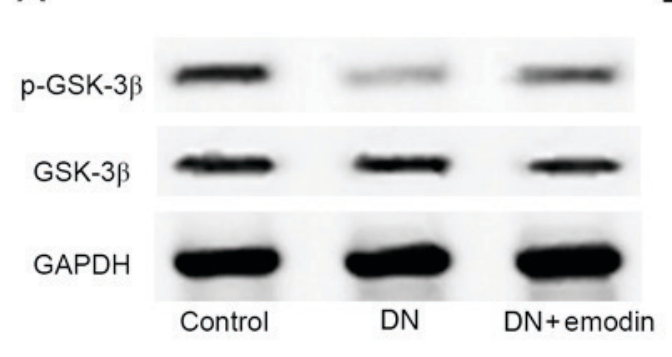

B

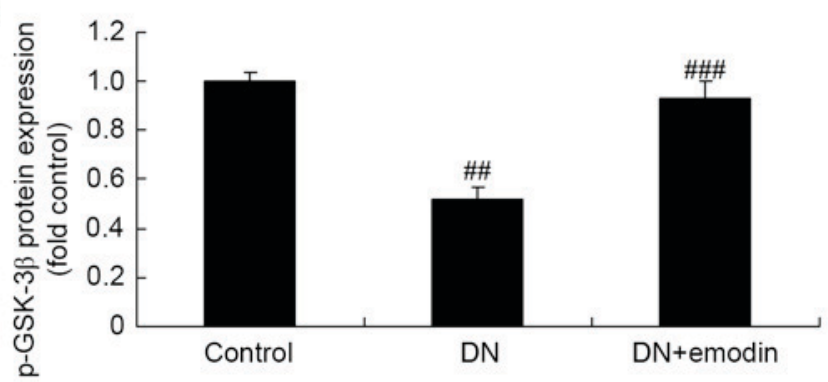

Figure 9. p-GSK-3b protein expression of diabetic rats. (A) Western blot analysis and (B) quantification of p-GSK-3ß protein expression in the kidneys of diabetic rats. ${ }^{\# \#} \mathrm{P}<0.01$ vs. the control group; ${ }^{\# \#} \mathrm{P}<0.01$ vs. the DM group. Control, control group; DN, diabetic nephropathy group; DN + emodin, diabetic nephropathy plus emodin treatment group; p-GSK-3 $\beta$, phosphorylated-glycogen synthase kinase 3.
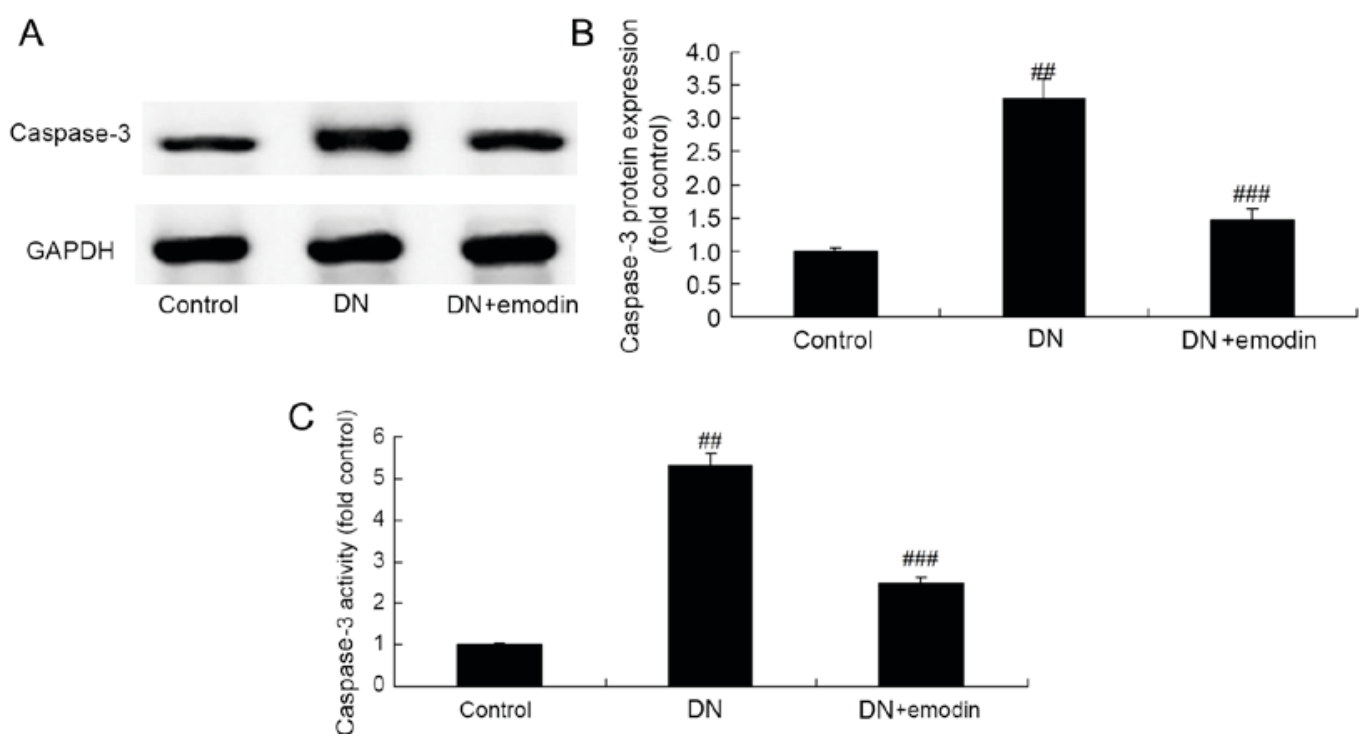

Figure 10. Caspase-3 in diabetic rats. (A) Western blot analysis and (B) quantification of caspase- 3 protein expression, and (C) ELISA results for caspase-3 in the kidneys of diabetic rats. ${ }^{\# \#} \mathrm{P}<0.01$ vs. the control group; ${ }^{\# \#} \mathrm{P}<0.01$ vs. the DM group. Control, control group; DN, diabetic nephropathy group; DN + emodin, diabetic nephropathy plus emodin treatment group.

the secretion of adhesion molecules, chemokine factors and cytokines are increased (19). Furthermore, a large number of immune cells gather and infiltrate into the kidney tissue, resulting in a further release of pro-inflammatory cytokines and growth factors, thereby increasing the inflammatory response and progressively exacerbating renal tissue damage and renal stromal fibrosis (20).
Previous studies have shown that the interaction between inflammation and DN includes complex molecular and pathway networks, and confirm the important role of inflammatory pathways in DN $(17,21)$. The results of the present study demonstrate the ability of emodin to significantly inhibit the blood glucose level, reduce the normalized kidney weight, and inhibit urinary albumin excretion and serum creatinine levels in 
rats DN. Furthermore, it decreased TII scores, attenuated the increased IL- 6 and TNF- $\alpha$ levels, elevated SOD activity and inhibited the MDA level in DN rats. Zeng et al (12) reported that emodin reduced uremic toxins in rats with chronic kidney disease. Zhu et al (14) demonstrated that emodin suppressed the LPS-induced inflammation of RAW264.7 cells and Xue et al (22) suggested that emodin attenuated lung injury through the suppression of oxidative damage in a mouse model.

ICAM-1 forms molecular bonds by the mutual contact between intermediate cells or between the cell and matrix. The majority of bonding sites comprise glycoproteins that are located on the cell surface or the extracellular matrix. Furthermore, ICAM-1 is expressed on a variety of cells, including endothelial and epithelial cells, fibroblasts, activated T cells, monocytes, macrophages and natural killer cells amongst others, which can enhance the adhesion between monocyte-macrophage and endothelial cells and cause inflammation (23). A continuously increased expression of ICAM-1 can cause serious damage to the structure and function of tissues and organs (24). Furthermore, ICAM-1 molecules are widely present in the body and are expressed in a variety of hematopoietic and non-hematopoietic cells, which are distributed mainly in various epithelial and endothelial cells, fibroblasts, reticular cells, monocytes, macrophages and lymphocytes (25). In the present study, it was revealed that emodin significantly suppressed ICAM-1 expression in DN model rats. Consistent with this, Chen et al (15) previously reported that emodin ameliorated LPS-induced corneal inflammation and ICAM-1 in rats.

The PI3K/Akt signaling pathway is involved in cell differentiation, proliferation, apoptosis and migration, and excessive activation of this pathway leads to cell dysfunction (26). A previous study indicated that PI3K/Akt signaling pathway activation occurs in association with DN, and high sugar or transforming growth factor- $\beta 1$ levels can cause changes to certain podocyte proteins such as ZO-1 and CD2AP by activating this pathway (27). Glomerular p-Akt expression in patients with DN has been observed to be increased with aggravation of the disease, although in severe lesions its expression is decreased and the expression of its negative regulation gene PTEN is gradually decreased with the increase of glomerular lesions; these results suggest that the PI3K/Akt signaling pathway is important in the development of early DN lesions $(10,27)$. The present study found that emodin significantly promoted $\mathrm{p}$-Akt protein expression in the rats with DN. Park et al (28) previously demonstrated that emodin induces the neurite outgrowth of Neuro2a cells through PI3K/Akt/GSK-3 $\beta$ signaling.

Among the factors associated with the insulin signal transduction pathway, GSK-3 $\beta$ has received considerable attention because of its multiple substrates and broad impact (29). For example, insulin receptor substrate-1 (IRS-1) results in a reduction in insulin receptor signaling following phosphorylation by GSK-3 $\beta$ (30). Cell experiments involving GSK-3 $\beta$ overexpression demonstrated that GSK-3 $\beta$ phosphorylates the serine and threonine residues of IRS-1, prevents tyrosine residues from undergoing phosphorylation by the insulin receptor, and reduces the level of IRS-1, thus weakening the insulin signaling pathway (30). The phosphorylation of cyclin D1 protein Thr268 by GSK-3 $\beta$ adjust the intracellular distribution and turnover of cyclin D1 (30). Furthermore, the axin and adenomatous polyposis genes may also be phosphorylated by GSK-3 $\beta(30,31)$.
Proteins associated with multiple signal transduction systems are regulated by GSK-3 $\beta$ phosphorylation, including transcription factors (10); numerous transcription factors are substrates of GSK-3 $\beta$, and are directly phosphorylated by GSK-3 $\beta$. The present study showed that emodin significantly increased the expression of p-GSK-3 $\beta$ and inhibited caspase- 3 activity and protein expression in rats with DN. A previous study conducted by $\mathrm{Wu}$ et al (32) demonstrated that emodin protected against diabetic cardiomyopathy through the KT/GSK-3 $\beta$ signaling pathway.

In conclusion, the results of the present study demonstrated that emodin significantly improved the average blood glucose levels, normalized kidney weight, urinary albumin excretion, serum creatinine levels and TII scores of diabetic rats. Furthermore, the underlying mechanism may be associated with anti-inflammatory and anti-oxidative activity, the suppression of ICAM-1, Bax and caspase-3, and activation of the Akt/GSK-3 $\beta$ signaling pathway. However, this requires further investigation in future studies.

\section{References}

1. Saito I, Azuma K, Kakikawa T, Oshima N, Hanson ME and Tershakovec AM: A randomized, double-blind, placebo-controlled study of the effect of ezetimibe on glucose metabolism in subjects with type 2 diabetes mellitus and hypercholesterolemia. Lipids Health Dis 14: 40, 2015.

2. Koloverou E, Panagiotakos DB, Pitsavos C, Chrysohoou C, Georgousopoulou EN, Laskaris A and Stefanadis C; ATTICA Study group: The evaluation of inflammatory and oxidative stress biomarkers on coffee-diabetes association: Results from the 10-year follow-up of the ATTICA Study (2002-2012). Eur J Clin Nutr 69: 1220-1225, 2015.

3. Lu HJ, Tzeng TF, Liou SS, Da Lin S, Wu MC and Liu IM: Ruscogenin ameliorates diabetic nephropathy by its anti-inflammatory and anti-fibrotic effects in streptozotocin-induced diabetic rat. BMC Complement Altern Med 14: $110,2014$.

4. Miyamoto S, Shikata K, Miyasaka K, Okada S, Sasaki M, Kodera R, Hirota D, Kajitani N, Takatsuka T, Kataoka HU, et al: Cholecystokinin plays a novel protective role in diabetic kidney through anti-inflammatory actions on macrophage: Anti-inflammatory effect of cholecystokinin. Diabetes 61: 897-907, 2012.

5. Saiki S, Sasazawa Y, Imamichi Y, Kawajiri S, Fujimaki T, Tanida I, Kobayashi H, Sato F, Sato S, Ishikawa K, et al: Caffeine induces apoptosis by enhancement of autophagy via $\mathrm{PI} 3 \mathrm{~K} / \mathrm{Akt} / \mathrm{mTOR} / \mathrm{p} 70 \mathrm{~S} 6 \mathrm{~K}$ inhibition. Autophagy 7: 176-187, 2011.

6. Ribback S, Cigliano A, Kroeger N, Pilo MG, Terracciano L, Burchardt M, Bannasch P, Calvisi DF and Dombrowski F: $\mathrm{PI} 3 \mathrm{~K} / \mathrm{AKT} / \mathrm{mTOR}$ pathway plays a major pathogenetic role in glycogen accumulation and tumor development in renal distal tubules of rats and men. Oncotarget 6: 13036-13048, 2015.

7. Huang C, Lin MZ, Cheng D, Braet F, Pollock CA and Chen XM: KCa3.1 mediates dysfunction of tubular autophagy in diabetic kidneys via PI3k/Akt/mTOR signaling pathways. Sci Rep 6: 23884, 2016.

8. Liu H, Mi S, Li Z, Hua F and Hu ZW: Interleukin 17A inhibits autophagy through activation of PIK3CA to interrupt the GSK3B-mediated degradation of BCL2 in lung epithelial cells. Autophagy 9: 730-742, 2013.

9. Qiao G, Le Y, Li J, Wang L and Shen F: Glycogen synthase kinase- $3 \beta$ is associated with the prognosis of hepatocellular carcinoma and may mediate the influence of type 2 diabetes mellitus on hepatocellular carcinoma. PLoS One 9: e105624, 2014.

10. Lee YJ and Han HJ: Troglitazone ameliorates high glucose-induced EMT and dysfunction of SGLTs through PI3K/Akt, GSK-3 $\beta$, Snaill and $\beta$-catenin in renal proximal tubule cells. Am J Physiol Renal Physiol 298: F1263-F1275, 2010.

11. Li H, Yang T, Zhou H, Du J, Zhu B and Sun Z: Emodin Combined with nanosilver inhibited sepsis by anti-inflammatory protection. Front Pharmacol 7: 536, 2017. 
12. Zeng YQ, Dai Z, Lu F, Lu Z, Liu X, Chen C, Qu P, Li D, Hua Z, $\mathrm{Qu} \mathrm{Y}$ and Zou C: Emodin via colonic irrigation modulates gut microbiota and reduces uremic toxins in rats with chronic kidney disease. Oncotarget 7: 17468-17478, 2016.

13. Huang YQ, Huang GR, Wu MH, Tang HY, Huang ZS, Zhou XH, Yu WQ, Su JW, Mo XQ, Chen BP, et al: Inhibitory effects of emodin, baicalin, schizandrin and berberine on hefA gene: Treatment of Helicobacter pylori-induced multidrug resistance. World J Gastroenterol 21: 4225-4231, 2015.

14. Zhu T, Zhang W, Feng SJ and Yu HP: Emodin suppresses LPS-induced inflammation in RAW264.7 cells through a PPAR $\gamma$-dependent pathway. Int Immunopharmacol 34: 16-24, 2016.

15. Chen GL, Zhang JJ, Kao X, Wei LW and Liu ZY: Emodin ameliorates lipopolysaccharides-induced corneal inflammation in rats. Int J Ophthalmol 8: 665-669, 2015

16. Li F, Lei T, Xie K, Wu X, Tang C, Jiang M, Liu J, Luo E and Shen G: Effects of extremely low frequency pulsed magnetic fields on diabetic nephropathy in streptozotocin-treated rats. Biomed Eng Online 15: 8, 2016.

17. Duran-Salgado MB and Rubio-Guerra AF: Diabetic nephropathy and inflammation. World J Diabetes 5: 393-398, 2014.

18. Wang JJ, Zhang SX, Mott R, Chen Y, Knapp RR, Cao W and Ma JX: Anti-inflammatory effects of pigment epithelium-derived factor in diabetic nephropathy. Am J Physiol Renal Physiol 294: F1166-F1173, 2008.

19. Baban B, Liu JY and Mozaffari MS: Endoplasmic reticulum stress response and inflammatory cytokines in type 2 diabetic nephropathy: Role of indoleamine 2,3-dioxygenase and programmed death-1. Exp Mol Pathol 94: 343-351, 2013

20. Bhattacharya S, Manna P, Gachhui R and Sil PC: D-saccharic acid 1,4-lactone protects diabetic rat kidney by ameliorating hyperglycemia-mediated oxidative stress and renal inflammatory cytokines via NF- $\kappa \mathrm{B}$ and PKC signaling. Toxicol Appl Pharmacol 267: $16-29,2013$

21. Chen S, Yang T, Liu F, Li H, Guo Y, Yang H, Xu J, Song J, Zhu Z and Liu D: Inflammatory factor-specific sumoylation regulates $\mathrm{NF}-\kappa \mathrm{B}$ signalling in glomerular cells from diabetic rats. Inflamm Res 63: 23-31, 2014.

22. Xue WH, Shi XQ, Liang SH, Zhou L, Liu KF and Zhao J: Emodin attenuates cigarette smoke induced lung injury in a mouse mode via suppression of reactive oxygen species production. J Biochem Mol Toxicol 29: 526-532, 2015.
23. Jain SK, Croad JL, Velusamy T, Rains JL and Bull R: Chromium dinicocysteinate supplementation can lower blood glucose, CRP, MCP-1, ICAM-1, creatinine, apparently mediated by elevated blood vitamin $\mathrm{C}$ and adiponectin and inhibition of NFkappaB Akt, and Glut-2 in livers of zucker diabetic fatty rats. Mol Nutr Food Res 54: 1371-1380, 2010.

24. Su X, Chen X, Liu L, Chang X, Yu X and Sun K: Intracellular adhesion molecule-1 K469E gene polymorphism and risk of diabetic microvascular complications: A meta-analysis. PLoS One 8: e69940, 2013

25. Tang LQ, Ni WJ, Cai M, Ding HH, Liu S and Zhang ST: The renoprotective effects of berberine and its potential impact on the expression of beta-arrestins and ICAM-1/VCAM-1 in streptozocin induced-diabetic nephropathy rats. J Diabetes 8: 693-700, 2016.

26. Dong M, Yang G, Liu H, Liu X, Lin S, Sun D and Wang Y: Aged black garlic extract inhibits HT29 colon cancer cell growth via the PI3K/Akt signaling pathway. Biomed Rep 2: 250-254, 2014.

27. Wang XM, Yao M, Liu SX, Hao J, Liu QJ and Gao F: Interplay between the Notch and PI3K/Akt pathways in high glucose-induced podocyte apoptosis. Am J Physiol Renal Physiol 306: F205-F213, 2014.

28. Park SJ, Jin ML, An HK, Kim KS, Ko MJ, Kim CM, Choi YW and Lee YC: Emodin induces neurite outgrowth through PI3K/Akt/GSK-3beta-mediated signaling pathways in Neuro2a cells. Neurosci Lett 588: 101-107, 2015.

29. Mariappan MM,Prasad S, D'Silva K, Cedillo E, Sataranatarajan K, Barnes JL, Choudhury GG and Kasinath BS: Activation of glycogen synthase kinase $3 \beta$ ameliorates diabetes-induced kidney injury. J Biol Chem 289: 35363-35375, 2014.

30. Shang G, Tang X, Gao P, Guo F, Liu H, Zhao Z, Chen Q, Jiang T, Zhang $\mathrm{N}$ and $\mathrm{Li} \mathrm{H}$ : Sulforaphane attenuation of experimental diabetic nephropathy involves GSK-3 beta/Fyn/Nrf2 signaling pathway. J Nutr Biochem 26: 596-606, 2015.

31. Singh LP, Jiang Y and Cheng DW: Proteomic identification of 14-3-3zeta as an adapter for IGF-1 and Akt/GSK-3beta signaling and survival of renal mesangial cells. Int J Biol Sci 3: 27-39, 2007.

32. Wu Z, Chen Q, Ke D, Li G and Deng W: Emodin protects against diabetic cardiomyopathy by regulating the AKT/GSK-3beta signaling pathway in the rat model. Molecules 19: 14782-14793, 2014. 